\title{
Basilar artery flow characteristics and color Doppler sonography findings in healthy infants
}

\author{
๑DHasibe Gökçe Çınar, ĐBerna Uçan \\ University of Health Sciences, Dr. Sami Ulus Training and Research Hospital, Department of Pediatric Radiology, Ankara, Turkey
}

Cite this article as: Çınar HG, Uçan B. Basilar artery flow characteristics and color Doppler sonography findings in healthy infants. J Health Sci Med 2022; 5(1): 173-177.

\begin{abstract}
Aim: Transcranial Doppler (TCD) is a widely used method for the evaluation of vertebrobasilar system. There is not neither a consensus nor significant number of publications about normal spectral Doppler waveform and resistance index (RI), peak systolic velocity (PSV), and end diastolic velocity (EDV) values of basilar artery (BA). We aim to define normal PSV, EDV, RI values of BA via TCD in healthy infants.

Material and Method: BA was evaluated from anterior fontanelle by creating sagittal and coronal images. Color Doppler ultrasonography (CDUS) and spectral Doppler examinations were performed by placing the cursor in the middle portion of BA. PSV, EDV, and RI values were recorded. Patients were divided into 4 subgroups to analyze the change of normal values according to age groups: (1) 0-120 days, (2) 121-180 days, (3) 181-270 days, (4) >271 days.

Results: 115 healthy infants were included into the study. A weak positive correlation was found between PSV, EDV values and age; meanwhile a weak but significant negative correlation was present between age and RI values. We cannot find any correlation between sex and CDUS parameters.

Conclusion: BA PSV, EDV and RI values change by age. No correlation is present between CDUS characteristics and sex. BA pathologies are rare in pediatric population, nevertheless knowing normal CDUS characteristics can help radiologists for an appropriate assessment.
\end{abstract}

Keywords: Basilar artery, blood flow, Doppler ultrasonography, transcranial Doppler ultrasound, infant

\section{INTRODUCTION}

Transcranial Doppler ultrasound or sonography (TCD) is main evaluation method of vertebrobasilar system and circle of Willis in pediatric patients before closing anterior and posterior fontanelles $(1,2)$. Both color Doppler ultrasound (CDUS) and spectral Doppler examinations can be performed transcranially $(3,4)$. Normal CDUS characteristics and spectral Doppler waveforms of many intracranial vascular structures such as common, internal/external carotid arteries, vertebral arteries and intracerebral arteries etc. were previously defined (5-7).

Basilar artery (BA) pathologies are not very common in infants. The main diagnosis in the pediatric population (between the ages of 0-17 years old) resulting from the basilary artery is mostly stroke. In these cases, the diagnosis was generally taken with computed tomographic angiography and/or conventional angiography (8-10). In literature, the knowledge about TCD usage for the evaluation of BA pathologies is very limited. In the same way, during our daily practice, we have noticed that a limited number of radiologists evaluate BA waveform during routine TCD. Also, as far as we know, there is not neither a consensus nor significant number of publications about normal spectral Doppler waveform and resistance index (RI), peak systolic velocity (PSV), and end diastolic velocity (EDV) values of BA.

Our study aims to define normal PSV, EDV, RI values of $B A$ via TCD in healthy infants and contribute a limited information repository.

\section{MATERIAL AND METHOD}

This current prospective study was carried out with the permission of Keçiören Training and Research Hospital Ethics Committee (Date: 20.06.2018, Decision No: 2012KAEK -11/2238). Informed consent form was signed by the parents of all participants. The study data was collected between February 2019 and August 2019. 
We performed TCD for examining BA to the infants who were referred to pediatric radiology clinic for a transcranial ultrasound examination. They were all healthy children whose parents were willing to participate, or the children came for another health issues: such as developmental dysplasia of hip, diarrhea, vomiting etc. We excluded patients with genetic and metabolic disease, heart failure, intracranial bleeding, or infection. Totally, 115 infants were included into the study.

CDUS examinations were performed with $3.5 \mathrm{MHz}$ convex transducers in longitudinal and transverse planes (iU22 Philips Healthcare, Best, the Netherlands; and Aplio, Toshiba Medical Systems, Japan).

BA was evaluated from anterior fontanelle by creating sagittal and coronal images. CDUS and spectral Doppler examinations were performed by placing the cursor in the middle portion of BA. PSV, EDV, and RI values were recorded (Figure 1). Patients were divided into 4 subgroups to analyze the change of normal values by age: (1) 0-120 days, (2) 121-180 days, (3) 181-270 days, (4) > 271 days.

\section{Statistical Analysis}

Data were analyzed using Statistical Package for Social Sciences (SPSS) 25 for Windows (IBM SPSS Inc., Chicago, IL). Normal distribution of the data was evaluated with the Kolmogorov-Smirnov test. Numerical were shown as median, minimum-maximum values. Categorical variables were shown as number and percentage. Consecutive variables were evaluated with Mann-Whitney $\mathrm{U}$ and Kruskal-Wallis tests. Spearman correlation analysis was used to define possible correlations between BA spectral Doppler parameters and age, sex.

\section{RESULTS}

Median age of the population was $69 \pm 2$ days (1-367 days). 69 participants (60\%) were males, 46 participants (40\%) were females.

Normal values of PSV, EDV, and RI values according to age subgroups can be seen in Table 1 and Figure 2. Median PSV, EDV, and RI values were found to be significantly different amongst age subgroups (Table 1, Kruskal-Wallis $\mathrm{p}$ value $=0.001$ ). According to Spearman correlation analysis results, a weak positive correlation was found between PSV, EDV values and age; meanwhile a weak but significant negative correlation was present between age and RI values (Table 2). We cannot find any correlation between sex and CDUS parameters.

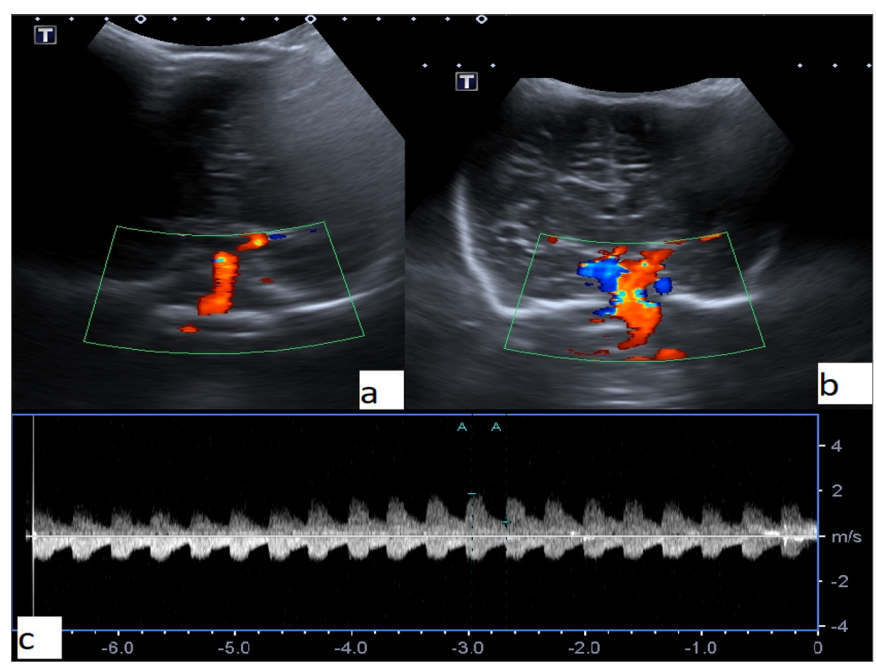

Figure 1. 153 days old healthy infant. CDUS images and spectral waveform acquired from basilar artery. PSV was $187.2 \mathrm{~cm} / \mathrm{s}$, EDV was $61.1 \mathrm{~cm} / \mathrm{s}$, RI was measured as 0.67 .

Table 1. Median Basilar artery PSV, EDV, and RI values according to age

\begin{tabular}{|cccccc|}
\hline Age (days) & Number & PSV $(\mathbf{c m} / \mathbf{s})$ & EDV $(\mathbf{c m} / \mathbf{s})$ & RI & P values $($ KW/MWU) \\
\hline $0-120$ & 75 & $113.4(95.7-397.2)$ & $30.2(11.7-122.3)$ & $0.71(0.7-0.89)$ & $P=0.001, P=0.03$ \\
$121-180$ & 22 & $153.3(13.1-204.3)$ & $53.2(3.4-82.4)$ & $0.62(0.6-0.79)$ & $P=0.001, P=0.04$ \\
$181-270$ & 14 & $164.8(13.5-248.4)$ & $75.8(7.8-119.7)$ & $0.54(0.51-0.72)$ & $P=0.001, P=0.001$ \\
271 and above & 4 & $206.6(186.8-247.2)$ & $86,7(77.9-101.1)$ & $0.58(0.54-0.59)$ & $P=0.001, P=0.02$ \\
Whole population & 115 & $131.9(95.7-397.2)$ & $44.8(11.7-122.3)$ & $0.66(0.7-0.89)$ & \\
\hline KW: Kruskal-Wallis, MWU: Mann- Whitney U, Note: Very low PSV, EDV and RI values were ignored in the table because these values are probably due to the crying effect. \\
\hline
\end{tabular}

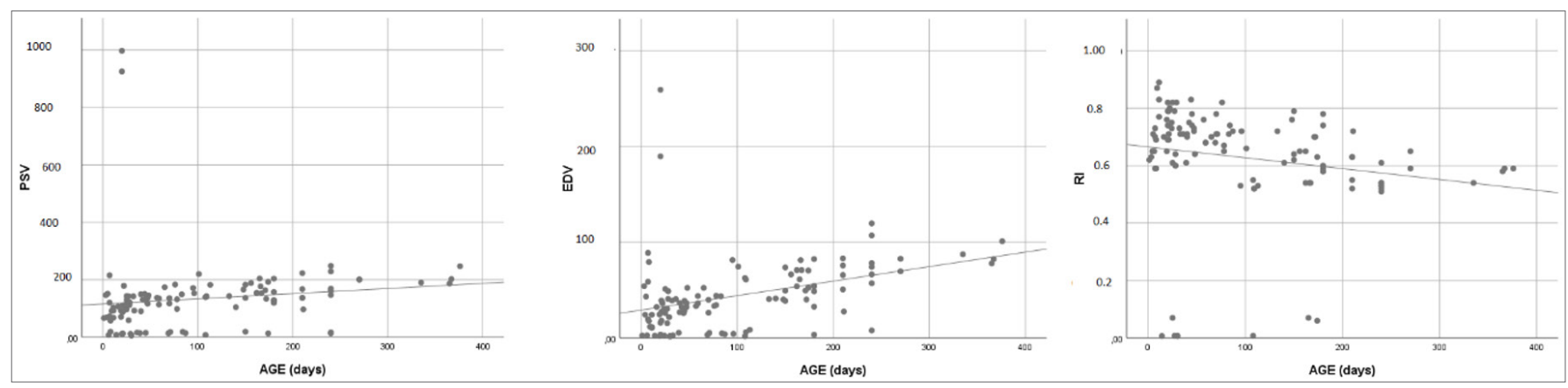

Figure 2. Scatterplots of median PSV, EDV, and RI values in healthy infant. 
Table 2. Correlations between age and PSV, EDV, RI values

\begin{tabular}{|lccc|}
\hline Correlations & PSV & EDV & RI \\
\hline \multirow{2}{*}{ Age } & $\mathrm{r}=0.45$ & $\mathrm{r}=0.49$ & $\mathrm{r}=(-) 0.42$ \\
& $\mathrm{p}=0.00$ & $\mathrm{p}=0.00$ & $\mathrm{p}=0.00$ \\
Sex & $\mathrm{r}=0.11$ & $\mathrm{r}=0.21$ & $\mathrm{r}=0.22$ \\
& $\mathrm{p}=0.07$ & $\mathrm{p}=0.1$ & $\mathrm{p}=0.09$ \\
\hline
\end{tabular}

\section{DISCUSSION}

In the current study, we defined the normal PSV, EDV, and RI values of basilar artery in newborn, neonate, and infants. We found that normal values of mentioned parameters change by age, and there was no statistically significant difference between genders $(p>0,05)$.

Blood flow of a vessel is affected from the difference of pressure between two ends of the vessel and the resistance of the vessel wall. Every major vessel has a characteristic CDUS pattern which is mainly created by PSV, EDV and RI values $(11,12)$.

CDUS is an important tool for the evaluation of major vessels in pediatric patients with lacking ionizing radiation, being easy to access and cheap etc. (13). Although the CDUS characteristics of major cranial arteries have been defined in the literature, there is not sufficient information about the basilar artery to the best of our knowledge $(5,6)$.

In the adult based study of Scheel et al. (14) if many studies compared, giving the name of the study will make it easy to understand), normal PSV value of BA was found as $131 \pm 21 \mathrm{~cm} / \mathrm{s}$. As far as we know, there was only one pediatric population-based study belongs to Binokay et al. (15) in the literature and according to the study's results, mean of PSV value was $84.7 \mathrm{~cm} / \mathrm{s}$. In our study, our median PSV value in the whole population was 131.1 $\mathrm{cm} / \mathrm{s}$. Our results were closer to the adult study than the pediatric study. The difference between the other pediatric population-based study and ours might be the result of populations' age characteristics; our median age was 69 days, mean age of the mentioned study was 10.2 years.

For vertebral artery, in neonates, PSV changes between $27-57 \mathrm{~cm} / \mathrm{sec}$ and PSV value of internal carotid artery is defined between $47-73 \mathrm{~cm} / \mathrm{s}(16,17)$. According to our data, median PSV value for the first 3 months of life was $131.1 \mathrm{~cm} / \mathrm{s}$, higher than vertebral and internal carotid arteries. The result is consistent with previous adultbased studies. Likewise, in adult-based studies, PSV values of BA are higher than PSV values of vertebral and internal carotid arteries $(18,19)$.

In the adult-based study of Scheel et al. (14) normal EDV value of BA was defined as $39.9 \pm 8.1 \mathrm{~cm} / \mathrm{s}$. Whereas, in the pediatric population-based study of Binokay et al., mean EDV value was $49.5 \mathrm{~cm} / \mathrm{s}$ (15). Our median normal EDV value was $39.1 \mathrm{~cm} / \mathrm{s}$, similar with adult values, slightly lower than the pediatric based study. The difference might be the result of populations' age characteristics, same as PSV values.

Two different studies belonging Kehrer et al. $(16,17)$ including neonatal population, EDV values of vertebral and internal carotid arteries were respectively altered at the range of $5-11 \mathrm{~cm} / \mathrm{s}$ and $13-21 \mathrm{~cm} / \mathrm{s}$. According to our results, EDV values of BA were higher than vertebral and internal carotid arteries. The results of another adultbased study of Schneel et al; EDV values of BA is also higher than EDV values of vertebral and carotid arteries like our study (19).

RI values can be calculated from PSV and EDV values via the formula (PSV-EDV)/PSV (20). In the literature, for adults, normal mean RI value of BA was defined as $0.58 \pm 0.10(16,17)$. For infants, mean RI value was $0.41 \pm 0.17$ (14).

According to the literature, the normal RI values of the internal carotid and vertebral arteries were ranged from 0.64 to 0.80 and from 0.73 to 0.89 , respectively, in the neonatal population $(17,18)$. Also, in full term neonates RI values of anterior, middle, and posterior cerebral arteries vary between 0.60 and 0.80 (9). These values were like our results (RI values of BA). We think that these similarities that do not change according to age population and the study also prove the reliability of our results.

According to a pediatric based study performed by Kehrer et al. (16) in healthy infants, EDV values of vertebral and internal carotid arteries increases with age. On the other hand, they cannot find any correlation between PSV values and age. In the same study, no significant correlation was defined between sex and CDUS parameters of vertebral/internal carotid arteries. As far as we know, there is no information about the correlation between CDUS parameters of BA and age/sex in the literature. Our results were partially consistent with the studies that discussed above. We cannot find any statistically correlation between sex and CDUS parameters $(\mathrm{p}>0,05)$. However, different from the literature, we defined a negative correlation between age and EDV, and a positive correlation between age and PSV. This subject is open for further prospective studies.

Considering the studies in the literature, flow velocities in all intracranial arteries decreased continuously from early childhood to adulthood and waveform parameters remained constant (21). This is probably due to the decrease in cerebrovascular resistance with age (22). In our study, arterial flow characteristics were found to be more like adults than children. 
This study has some limitations including the small population number. We did not have any participants older than 1 years of age. Our results include only a small part of the pediatric population. Interobserver variability of CDUS parameters was ignored since only a small number of infants was evaluated by both authors. We have studied only with healthy infant, further studies including the cases with BA pathologies might help to define cut off values for abnormal cases. The technical limitations of the CDUS examination of the pediatric population, such as patient agitation and difficulty creating optimal waveforms, were also valid for our study.

PSV and EDV rates were extremely low in several patients. To the best of our knowledge, it is not known the exact reason but crying could have been a factor, altering brain hemodynamics and misleading RDUS measurements. In healthy humans, the Valsalva maneuver causes characteristic changes in flow velocity in the cerebral arteries, reflecting sympathetic and cerebral autoregulation responses, respectively. Considering crying and Valsalva maneuver is similar mechanism, this hypothesis can explain changes in the parameters of cranial Doppler ultrasonography. All of the children in our study were younger than 1 year old and cried from time to time during the examination. This effect is another limitation of our study (23).

As we tried to summarize and emphasize, there is not sufficient information about CDUS characteristics of BA. There is only one pediatric population based study in the literature (8), and it has different results with regard to our study; possibly as a result of examining different age groups. We believe that it is obviously needed to conduct further prospective studies including wide range of age groups, to define normal CDUS parameter ranges more precisely and correctly.

\section{CONCLUSION}

BA PSV, EDV and RI values change by age. No correlation is present between CDUS characteristics and sex. BA pathologies are rare in pediatric population, nevertheless knowing normal CDUS characteristics can help radiologists for an appropriate assessment.

\section{ETHICAL DECLARATIONS}

Ethics Committee Approval: The study was carried out with the permission of Keçiören Training and Research Hospital Ethics Committee (Approval date and number: 20.06.2018/ 2012-KAEK -11/2238).

Informed Consent: Because the study was designed retrospectively, no written informed consent form was obtained from patients.

Referee Evaluation Process: Externally peer-reviewed. Conflict of Interest Statement: The authors have no conflicts of interest to declare.

Financial Disclosure: The authors declared that this study has received no financial support.

Author Contributions: All of the authors declare that they have all participated in the design, execution, and analysis of the paper, and that they have approved the final version.

\section{REFERENCES}

1. Kirsch JD, Mathur M, Johnson MH, Gowthaman G, Scoutt LM. Advances in transcranial Doppler US: imaging ahead. Radiographics 2013; 33: 1-14.

2. Verlhac S. Transcranial Doppler in children. Pediatr Radiol 2011; 41: 153-65.

3. The American College of Radiology. ACR-AIUM practice guideline for the performance of transcranial Doppler ultrasound for adults and children. Available at: http: //www.acr.org/ Secondary MainMenuCategories/quality_safety/guidelines/us/ us_transcranial_Doppler.aspx. (2007). Accessed 8 March 2011.

4. Schatlo B, Pluta RM. Clinical applications of transcranial Doppler sonography. Rev Recent Clin Trials 2007; 2: 49-57

5. Chavhan GB, Parra DA, Mann A, Navarro OM. Normal Doppler spectral waveforms of major pediatric vessels: specific patterns. Radiographics 2008; 28: 691-706.

6. Couture AP. Normal neonatal brain: Color Doppler and pulsed Doppler. In: Couture A, Veyrac C, eds. Transfontanellar Doppler İmaging in Neonates. Heidelberg, Germany: Springer, 2001; 9-90.

7. Carroll BA. The extracranial cerebral vessels. In: Rumack CM, Wilson SR, Charboneau JW, Johnson J, eds. Diagnostic ultrasound. 3rd ed. St. Louis, Mo: Elservier Mosby, 2005; 943-92.

8. Lagman-Bartolome AM, Pontigon AM, Moharir M, et al. Basilar artery strokes in children: good outcomes with conservative medical treatment. Dev Med Child Neurol 2013; 55: 434-9.

9. Lobbous M, Williams S, Rashid S. Child neurology: childhood basilar artery occlusion and stroke. Neurology 2017; 89: 68-70.

10. Chikkannaiah M, Lo WD. Childhood basilar artery occlusion: a report of 5 cases and review of the literature. J Child Neurol 2014; 29: 633-45.

11. Taylor KJ, Holland S. Doppler US. Part I. Basic principles, instrumentation, and pitfalls. Radiology 1990; 174: 297-307.

12. Rubens DJ, Bhatt S, Nedelka S, Cullinan J. Doppler artifacts and pitfalls. Radiol Clin North Am 2006; 44: 805-35.

13. Couture AP. Normal neonatal brain: colour Doppler and pulsed Doppler. In: Couture A, Veyrac C, eds. Transfontanellar Doppler imaging in neonates. Heidelberg, Germany: Springer, 2001; 9-90.

14. Scheel P, Ruge C, Petruch UR, Scho"ning M. Color duplex measurement of cerebral blood flow volume in healthy adults. Stroke 2000; 31: 147-50

15. Binokay F, Balli T, Aikimbaev K, Seydaoğlu G, Çiçekçi S, Yalçın Ç. Normal transcranial Doppler flow parameters in children: implementation of Doppler imaging with angle correction. Neurosurgery Quarterly 2015; 25: 6-11.

16.Kehrer M, Goelz R, Schoning M. The development of haemodynamics in the extracranial cerebral arteries of healthy preterm and term neonates. Ultrasound Med Biol 2004; 30: 2837. 
17. Kehrer M, Schoning M. A Longitudinal study of cerebral blood flow over the first 30 months. Pediatr Res 2009; 66: 560-4.

18. Yazici B, Erdogmus B, Tugay A. Cerebral blood flow measurements of the extracranial carotid and vertebral arteries with Doppler ultrasonography in healthy adults. Diagn Interv Radiol 2005; 11 : 195-8.

19. Scheel P, Ruge C, Schoning M. Flow velocity and flow volume measurements in the extracranial carotid and vertebral arteries in healthy adults: reference data and the effects of age. Ultrasound Med Biol 2000; 26: 1261-6.

20. Yang J, Hua Y, Li X, et al. The assessment of diagnostic accuracy for basilar artery stenosis by transcranial color-coded sonography. Ultrasound Med Biol 2018; 44: 995-1002.

21.Schöning M, Niemann G, Hartig B. Transcranial color duplex sonography of basal cerebral arteries: reference data of flow velocities from childhood to adulthood. Neuropediatrics 1996; 27: 249-55.

22.Bode $\mathrm{H}$,Wais U. Age dependence of flow velocities in basal cerebral arteries. Arch Dis Child 1988; 63: 606-11.

23. Perry BG, Cotter JD, Mejuto G, Mündel, T, Lucas SJE. Cerebral hemodynamics during graded Valsalva maneuvers. Front Physio 2014; 5: 349. 